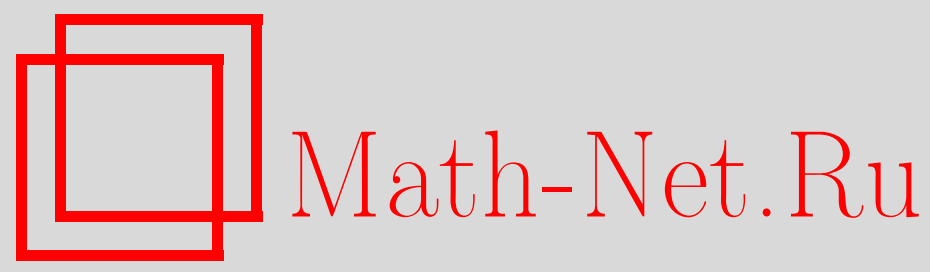

А. В. Лобода, Об определении однородной строго псевдовыпуклой гиперповерхности по коэффициентам ее нормального уравнения, Матем. заметки, 2003, том 73, выпуск $3,453-456$

DOI: https://doi.org/10.4213/mzm616

Использование Общероссийского математического портала Math-Net.Ru подразумевает, что вы прочитали и согласны с пользовательским соглашением http://www . mathnet.ru/rus/agreement

Параметры загрузки:

IP : 52.87 .193 .239

26 апреля 2023 г., 15:43:09

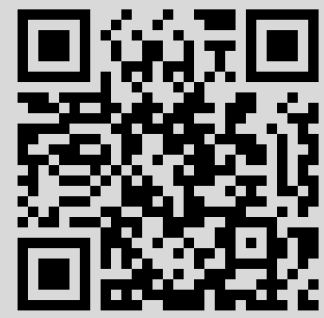




\section{ОБ ОПРЕДЕЛЕНИИ ОДНОРОДНОЙ СТРОГО ПСЕВДО-ВЫПУКЛОЙ ГИПЕРПОВЕРХНОСТИ ПО КОЭФФИЦИЕНТАМ ЕЕ НОРМАЛЬНОГО УРАВНЕНИЯ}

\section{А. В. Лобода}

1. Основные формулировки. В 3-мерном комплексном пространстве рассмотрим вещественную гиперповерхность, локально однородную относительно голоморфных преобразований. Везде в статье локальная однородность многообразия $M$ в некоторой его точке $P$ означает существование локальной группы Ли $G$, действующей на $M$ транзитивно вблизи точки $P$. При этом элементами группы $G$ являются биголоморфные преобразования объемлющего $M$ пространства, определенные вблизи $P$.

Локально однородные гиперповерхности двумерных комплексных пространств были полностью описаны (в явной форме и в терминах соответствующих алгебр Ли) Э. Картаном в [1]. А в силу [2] локално однородная невырожденная по Леви (а значит, строго псевдо-выпуклая) гиперповерхность 2-мерного комплексного пространства определяется тейлоровскими коэффициентами своего нормального мозеровского (см. [3]) уравнения не более, чем восьмого порядка.

ТеОРема 1. Локально однородная строго псевдо-выпуклая гиперповерхность 3-мерного комплексного пространства определяется тейлоровскими коэффичиентами своего нормального уравнения не более, чем седьмого порядка.

Ниже приводятся два утверждения, уточняющие этот результат.

ТЕОРема 2. Локально однородная строго псевдо-выпуклая гиперповерхность 3-мерного комплексного пространства, имеющая более, чем дискретную группу изотропии, определяется тейлоровскими коэффициентами своего нормального уравнения не более, чем шестого порядка.

Напомним, что для группы изотропии $\operatorname{Aut}_{P}(M)$ однородной несферической строго псевдо-выпуклой гиперповерхности $M$ 3-мерного комплексного пространства выполняется (см. [4]) оценка $\operatorname{dim} \operatorname{Aut}_{P}(M) \leqslant 2$.

Полный список однородных поверхностей, имеющих в точности 2-мерные группы изотропии, построен в [5].

Теорема 3. Все локально-однородные строго псевдо-выпуклье вещественные гиперповерхности 3-мерных комплексных пространств, имеющ,ие 1-мерные әруппы изотропии, задаются с точностью до локальной голоморфной эквивалентности следующим списком попарно неэквивалентных однородных поверхностей пространства $\mathbb{C}^{3}\left(z_{1}=x_{1}+i y_{1}\right.$,

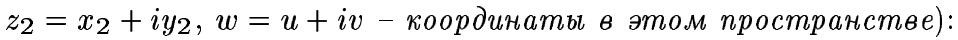

$$
\begin{aligned}
& v=x_{2}^{2}+\left(\left(1+x_{1}\right)^{\alpha}-1\right), \quad \alpha \in(-\infty, 0) \cup(1,2) \cup(2, \infty), \\
& v=x_{2}^{2}-\left(\left(1+x_{1}\right)^{\alpha}-1\right), \quad \alpha \in(0,1), \\
& v=x_{2}^{2}+\left(1+x_{1}\right) \ln \left(1+x_{1}\right), \\
& x_{1}^{2}+x_{2}^{2}+u^{2}=1, \\
& \quad-x_{1}^{2}-x_{2}^{2}+u^{2}=1, \\
& 1 \pm\left(\left|z_{1}\right|^{2}+\left|z_{2}\right|^{2}\right)+|w|^{2}=a\left|1+z_{1}^{2}+z_{2}^{2}+w^{2}\right|, \quad a>1, \\
& 1 \pm\left(\left|z_{1}\right|^{2}+\left|z_{2}\right|^{2}\right)-|w|^{2}=a\left|1+z_{1}^{2}+z_{2}^{2}-w^{2}\right|, \quad 0<a<1 .
\end{aligned}
$$

Работа выполнена при поддержке Российского фонда фундаментальных исследований, гранты № 01-01-00594 и № 99-01-00969, а также Программы поддержки ведущих научньг шшкол, грант № 0015-06-008. 
2. Схема доказательства. Для доказательства теорем 1-3 рассматривается алгебра g голоморфных векторных полей, касательных к однородной поверхности $M$. Для любого такого поля $Z$, имеющего в координатах вблизи $M=\left\{\Phi\left(z_{1}, z_{2}, w\right)=0\right\}$ вид

$$
Z=f^{(1)}(z, w) \frac{\partial}{\partial z_{1}}+f^{(2)}(z, w) \frac{\partial}{\partial z_{2}}+g(z, w) \frac{\partial}{\partial w}
$$

выполняется тождество

$$
\left.\operatorname{Re}\{Z(\Phi)\}\right|_{M} \equiv 0 .
$$

Задавая вещественно-аналитическую поверхность $M$ нормалным в смысле Мозера уравнением

$$
v=\left(\left|z_{1}\right|^{2}+\left|z_{2}\right|^{2}\right)+\sum_{k, l \geqslant 2, m \geqslant 0} h_{k l m}(z, \bar{z}) u^{m}
$$

и учитывая голоморфность $f^{(1)}, f^{(2)}, g$, рассмотрим левую часть (8) как аналитическую функцию от пяти вещественных переменных $z_{1}, \bar{z}_{1}, z_{2}, \bar{z}_{2}, u$.

Обозначая через $(k, \bar{l}, m)$ набор степеней соответственно по переменным $z, \bar{z}, u$, будем рассматривать $(k, \bar{l}, m)$-компоненты основного тождества $(8)$.

Учтем при этом следующее уточнение резултата [3] (см. еще [6]).

ПРЕДЛОЖЕНИЕ 1. Многочлен $h_{220}$ из нормального уравнения (9) несферической поверхности $M$ мохно привести к одному из двух видов:

$$
h_{220}= \pm E_{0},
$$

uлu

$$
h_{220}= \pm\left(a E_{0}+E_{3}\right), \quad a \geqslant 0,
$$

əде $E_{0}=\left(\left|z_{1}\right|^{4}-\left.4\left|z_{1}\right|^{2}\left|z_{2}\right|^{2}|+| z_{2}\right|^{4}\right), E_{3}=\left(\left|z_{1}\right|^{2}-\left|z_{2}\right|^{2}\right)\left(z_{1} \bar{z}_{2}+z_{2} \bar{z}_{1}\right)$.

Из $(2, \overline{2}, 0)$ - и $(3, \overline{2}, 0)$-компонент основного тождества получаем

ПРЕДЛОЖЕНИЕ 2. Если в нормальном уравнении (9) однородной поверхности $M$ многочлен $h_{220}$ имеет вид (11), то группа изотропии $M$ мохет быть только дискретной.

В этом случае размерность алгебры g равна размерности самой поверхности $M$, т.е. 5 . При этом координатами в алгебре $\mathfrak{g}$ являются

$$
p_{1}=f^{(1)}(0,0) \in \mathbb{C}, \quad p_{2}=f^{(2)}(0,0) \in \mathbb{C}, \quad q=g(0,0) \in \mathbb{R},
$$

а компоненты $\left(f^{(1)}, f^{(2)}, g\right)$ любого поля $Z$ из этой алгебры линейно выражаются через $(p, \bar{p}, q)$.

Тогда любая $(k, \bar{l}, m)$-компонента тождества (8) (помимо уже рассмотренных) имеет вид

$$
q h_{k, l, m+1}+\partial h_{k+1, l, m}(p)+\bar{\partial} h_{k, l+1, m}(\bar{p})=\varphi(p, \bar{p}, q)
$$

где правая часть линейна по $p, \bar{p}, q$ и выражается через многочлены $h_{s t r}$ из нормального уравнения (9) с меньшими весами $(s+t+2 r)$.

ЛЕмма (о единственности). Уравнение (12), выполняющееся тождественно по $p, \bar{p}, q$, позволяет выразить $h_{k, l, m+1}, h_{k+1, l, m}, h_{k, l+1, m}$ через многочлены $h_{s t r}$ из правой часmu.

Индукция по весу и использование леммы о единственности приводят в этом случае к следующему утверждению.

ПРЕДЛОЖЕНИЕ 3. Если в нормальном уравнении (9) однородной поверхности многочлен $h_{220}$ приводится $\kappa$ виду (11), то все многочлены $h_{k l m}$ из этого уравнения однозначно определяются набором

$$
J=\left\{h_{220}, h_{221}, h_{222}, h_{320}, h_{321}, h_{420}, h_{330}\right\} .
$$


ЗАмечАниЕ. Многочлены опорного набора (13) формируются из тейлоровских коэффициентов уравнения (9) не более, чем шестого порядка.

Рассмотрим теперь многочлен $h_{220}$ вида (10). Здесь справедливо

ПРЕДЛОЖЕНИЕ 4. Если $h_{220}=\varepsilon E_{0}(\varepsilon= \pm 1)$ и однородная поверхность $M$ имеет более, чем дискретную группу изотропии, то многочлен $h_{320}$ из ее нормального уравнения (9) мохсно привести к виду

$$
h_{320}=\omega_{1}\left(z_{1}^{3} \bar{z}_{1}^{2}-2 z_{1}^{2} z_{2} \bar{z}_{1} \bar{z}_{2}-z_{1} z_{2}^{2} \bar{z}_{2}^{2}\right), \quad \omega_{1} \geqslant 0 .
$$

В рамках (14) мы обсудим два случая. В первом из них считаем коэффициент $\omega_{1}$ строго положительным.

ПРЕДЛОЖЕНИЕ 5. Если нормальное уравнение (9) однородной поверхности М удовлетворяет условиям

$$
h_{220}=\varepsilon E_{0}, \quad h_{320}=\omega_{1}\left(z_{1}^{3} \bar{z}_{1}^{2}-2 z_{1}^{2} z_{2} \bar{z}_{1} \bar{z}_{2}-z_{1} z_{2}^{2} \bar{z}_{2}^{2}\right), \quad \omega_{1}>0,
$$

mo

а) все многочлены $h_{k l m}$ из нормального уравнения выражсатся через опорный набор многочленов $J_{1}=\left\{h_{220}, h_{320}, h_{420}\right\}$;

б) $h_{420}=s_{1} z_{1}^{4} \bar{z}_{1}^{2}$ и коэффичиенты $\varepsilon, \omega_{1}, s_{1}$ опорного набора связаны соотношением

$$
3 \varepsilon \omega_{1}^{4}-7 s_{1} \omega_{1}^{2}+4 \varepsilon s_{1}^{2}-12 \omega_{1}^{2}=0 .
$$

Для однородных поверхностей из второго случая в (14), т.е. при

$$
h_{220}=\varepsilon\left(\left|z_{1}\right|^{4}-4\left|z_{1}\right|^{2}\left|z_{2}\right|^{2}+\left|z_{2}\right|^{4}\right), \quad h_{320}=0
$$

из $(3, \overline{2}, 0)$-компоненты основного тождества следует, что многочлен

$$
h_{420}=s_{8}\left(z_{1}^{3} z_{2} \bar{z}_{1}^{2}+z_{1}^{2} z_{2}^{2} \bar{z}_{1} \bar{z}_{2}+z_{1} z_{2}^{3} \bar{z}_{2}^{2}\right), \quad s_{8} \geqslant 0 .
$$

Изучение $(2, \overline{2}, 1)-,(4, \overline{2}, 0)-,(3, \overline{3}, 0)-,(3, \overline{2}, 1)$-компонент при условии $s_{8}=0$ приводит к однородным поверхностям с 2-мерными группами изотропии (см. [5]). Каждая из них определяется парой многочленов $h_{220}, h_{330}$.

При $s_{8} \neq 0$ получаем

ПРЕДЛОЖЕНИЕ 6. Если нормальное уравнение (9) однородной поверхности М удовлетворяет условиям

$$
\begin{gathered}
h_{220}=\varepsilon E_{0}, \quad h_{320}=0, \\
h_{420}=s_{8}\left(z_{1}^{3} z_{2} \bar{z}_{1}^{2}+z_{1}^{2} z_{2}^{2} \bar{z}_{1} \bar{z}_{2}+z_{1} z_{2}^{3} \bar{z}_{2}^{2}\right), \quad s_{8}>0,
\end{gathered}
$$

то все многочлены $h_{k l m}$ из ее нормального уравнения выражсатся через опорный набор многочленов $J_{2}=\left\{h_{220}, h_{420}\right\}$.

Завершается описание однородных поверхностей с 1-мерными группами изотропии ссылкой на следуюшие технические факты.

ПРЕДЛОЖЕНИЕ 7. Нормальные мозеровские уравнения однородных трубчатых поверхностей (1)-(3) относятся $к$ случало (15). При этом

$$
\varepsilon=\operatorname{sgn}(\alpha-2), \quad \omega_{1}=\frac{\sqrt{24} \alpha}{\sqrt{|\alpha-2|}}, \quad s_{1}=\frac{-12 \alpha(2 \alpha-1)}{\alpha-2}
$$

для поверхностей (1) и (2), а для поверхности (3) эти жее формуль справедливы при $\alpha=1$. 
ПРЕДЛОЖЕНИЕ 8. Нормальные мозеровские уравнения однородных поверхностей (4)-(7) относятся $к$ случаю (18). При этом

а) $s_{8}=9 a$ для поверхностей (6), (7) и $s_{8}= \pm 9$ для трубок над сферой и зиперболоидом;

б) параметр $\varepsilon= \pm 1$ из формул (6), (7) является коэффичиентом многочлена $h_{220}$ из формуль (10).

Непосредственно проверяется, что наборы (19) исчерпьвают все возможности, предоставляемые формулой (16) и неравенством $\omega_{1}>0$. Аналогично, пара $\left(\varepsilon, s_{8}\right)$ из предложения 10 принимает любые значения, возможные в рамках случая (18). Кроме того, все поверхности (1)-(7) имеют 1-мерные группы изотропии (например, уравнение (1) легко превращается в новое уравнение $v=\left|z_{2}\right|^{2}+\left(\left(1+x_{1}\right)^{\alpha}-1\right)$, инвариантное относительно поворотов комплексной переменной $\left.z_{2}\right)$.

Теоремы 2 и 3 , тем самьм, доказаны.

Для доказательства теоремы 1 вернемся к нерассмотренным возможностям, связанным с многочленом $h_{220}$ вида (10). Помимо случая (15) здесь представляет интерес возможность

$$
h_{220}=\varepsilon E_{0}, \quad h_{320}=\omega_{2} z_{1}^{3} \bar{z}_{1} \bar{z}_{2}, \omega_{2}>0 .
$$

ПРЕДЛОЖЕНИЕ 9. Если нормальное уравнение (9) однородной поверхности $M$ не сводится к случаям (15) или (20), то из $(3, \overline{2}, 0)$-тохдества для такой поверхности следует, что все многочлень $h_{k l m}$ из нормального уравнения однозначно определяются тейлоровскими коэффициентами этого уравнения не более, чем шестого порядка.

В случае (20) ситуация оказьвается несколько более сложной.

ПРЕДЛОЖЕНИЕ 10. Если нормальное уравнение (9) однородной поверхности М удовлетворяет условиям (20), то из $(3, \overline{2}, 0)-,(2, \overline{2}, 1)-,(4, \overline{2}, 0)$-тохсеств для такой поверхносmи следует, что все многочлены $h_{k l m}$ из нормального уравнения однозначно определяются тейлоровскими коэффициентами этого уравнения не более, чем седьмого порядка.

\section{СПИСОК ЦИТИРОВАННОЙ ЛИТЕРАТУРЫ}

1. Cartan E. // Ann. Math. Pura Appl. (4). 1932. V. 11. P. 17-90; // Oeuvres II. V. 2. P. 12311304. 2. Л обода А. В. / Функцион. анализ и его прил. 2000. Т. 34. № 2. C. 33-42. 3. Chern S. S., Moser J. K. // Acta Math. 1974. V. 133. № 3. P. 219-271. 4. Лобода А. В. // Функцион. анализ и его прил. 1999. Т. 33. № 1. С. 68-71. 5. Лобода А. В. // Матем. сб. 2001. Т. 192. № 12. С. 3-24. 6. Ежов В. В., Лобода А. В., Шмальц Г. // Матем. заметки. 1999. Т. 66. № 4. С. 624-626. 